\title{
UTILIZATION OF CLOUD COMPUTING (MICROSOFT AZURE) FOR ONLINE BUSINESS
}

\author{
Rizki Amalia \\ Study Program Informatic Technique \\ Majoring in Informatics and Computer Technology \\ Politeknik Negeri Jakarta \\ Depok, West Java, Indonesia \\ rizkiamalia558@gmail.com
}

\begin{abstract}
Cloud computing has been a disruptive innovation in recent years and has made headlines to replace many established traditional computing methodologies in a short time. This essay will investigate the technical aspects of cloud computing and the strategic and competitive advantages it brings to companies at startup. Although many have criticized this technology business model because of concerns about security, privacy and governance, recent events prove that more entrants, including company leaders in the industry, enter the cloud and make their presence felt by customers and Microsoft Azure offers a data center that are reliable, available and maintainable with the right funds to build a data center that can solve this problem.
\end{abstract}

Keywords: Cloud Computing, Microsoft Azure

\section{INTRODUCTION}

Did you know what is Cloud computing ? Cloud computing is the mode of executing the IT services in an elastic manner to the end users and providing a metered service at multiple granularities for a specified quality of service. Gartner defines cloud computing as "a style of computing where massively scalable IT-related capabilities are provided 'as a service' using Internet technologies to multiple external customers". While Forrester says "Cloud computing is a new IT outsourcing model that doesn't yet meet the criteria of enterprise IT and isn't supported by most of the key corporate vendors. It's wildly popular with startups, exactly fits the way small businesses like to buy things, and has the potential to completely upend IT as we know it."

Microsoft Azure is a cloud computing platform that offers PaaS (Platform as a service) services. You can use this platform to build a powerful web-based application. In addition, Azure has a hosting platform service with an on-demand payment scheme, so we can manage storage, bandwidth and other needs as we please. Then the payment also uses the pay-as-you-go concept. That is, whatever resources we have used will be clearly calculated when billing or billing cycle.

Cloud sophistication is a service and its resources are very strong and strong. It is very unlikely to fail to run because of smart cloud and can do recovery independently. With Azure, there are data centers around the world, which are full of tens of thousands of servers.

If one server fails, another server will take over. If the entire data center fails (an impossible 
scenario), another data center will take over. All this is possible because of the huge scale of the cloud. In fact, Microsoft provides Service Level Agreements (SLAs) that promise services will stay active and running or you can ask for money back. Azure provides one of the highest SLAs in the industry for its services.

One of the most interesting arguments that supports the cloud is that you don't need to have a server anymore. This means that you are not responsible for buying it and keeping it running. In addition, you don't need to worry about network configuration from setting up your server. It is all done for you - including ensuring that the server remains cool and safe and pays electricity bills.

In addition, you can improve services and resources almost unlimited with just a few clicks on the button. This cannot be done with local sources unless you are ready to spend large amounts of money on capital equipment and administrative staff. You can also increase the scale to global. You can place your services anywhere in the world so you can offer highlevel experience to your users, wherever they are. This also means you can store data where you need it. Equally important, when you use cloud resources, you can change the scale of your services and resources again when demand is not large.

\section{METHODOLOGY}

This research is categorized as literature research because it collects information from many other literature. This study collects data from many reliable sites.

Data for this study are articles taken from trusted sources. The research instrument is online media.

\section{RESULT AND DISCUSSION}

\section{Increase and Apply Disaster Reserves} and Recovery

According to Lodewijk, when traffic is high, Microsoft Azure allows its startup to automatically increase its scalability. When traffic is normal, scalability will also return to normal. This ability really helps Bhinneka to maximize customer convenience in making transactions.

As a cloud-based solution, Azure is innately flexible - it can back up your data in almost any language, on any OS, and from any location. Plus, you define the frequency and extent of your backup schedule (daily, weekly, monthly, etc.).

Tape backup has a time and place, but it has limited abilities as a stand-alone backup and disaster recovery solution. Azure site recovery can enhance your tape backup with offsite replication, minimal onsite maintenance, up to ninety-nine years of data retention, minimal or no capital investment, and minimal operational costs. Azure backup stores three copies of your data in three different locations in the data center, and then another three copies in a remote Azure data center, so you never have to worry about losing data.

"We pay for resources at Azure as needed because of this variety of e-commerce services so the nature of traffic is different. The most important thing for e-commerce business is to keep the business process running, especially when online payments must be recorded. That is the most important, "explained Lodewijk. 


\section{Host and Develop Web and Mobile Apps}

Whether you're looking for a platform for hosting, developing, or managing a web or mobile app, Azure makes those apps autonomous and adaptive with patch management, AutoScale, and integration for on-premise apps. With Automatic patch management for your virtual machines, you can spend less time managing your infrastructure and focus on improving your apps. Azure also comes with continuous deployment support, which allows you to streamline ongoing code updates.

AutoScale is a feature built into Azure Web Apps that adjusts your resources automatically based on customer web traffic so you have the resources you need when traffic is high, and save money when you're not in peak times.Through Azure, you can seamlessly link your web app to an on-premise app. Connecting apps in both locations lets both employees and partners securely access resources inside your firewall-resources that would otherwise be difficult to access externally.Costs incurred by his party to get all services from Microsoft Azure are claimed to be no more than $\$ 500$ per month.

\section{Distribute and Supplement Active Directory}

Azure can integrate with your Active Directory to supplement your identity and access capabilities - this gives your DNS a global reach, centralized management, and robust security.

With Azure, you can globally distribute an Active Directory environment that is direct connect enabled. No other cloud provider has the ability to extend the reach of your domain controller and consolidate AD management like Azure.
If you have multiple locations or use onpremise apps or cloud apps like Office 365, Active Directory integration with Azure will be the central tool for managing and maintaining access to all of these tools.

Azure also enables you to utilize multi-factor authentication, adding a new layer of security to your data and applications with zero hassle for your users. You can also easily implement single sign-on for Windows, Mac, Android, and iOS cloud apps.

\section{Innovate with IoT Industry Solutions}

The scalability, flexibility, and security of Microsoft Azure makes it the perfect resource for companies moving toward IoT solutions. You can connect your devices to the cloud using solutions that integrate with your existing infrastructure and start collecting new data about your company.

Within the Azure IoT Hub, you can monitor and manage billions of devices and gain insights to help you make better business decisions, improve customer experiences, reduce complexity, lower costs, and speed up development.

The enhanced security of Azure is a huge asset for IoT solutions, which traditionally have security gaps that hackers can take advantage of. Other benefits include remote monitoring and predictive maintenance and analytics.

Getting started is easy with Azure IoT solution accelerators, preconfigured templates that are customizable to your needs. 


\section{CONCLUSION}

These four services are just a glimpse of what Azure can do for your environment. Besides Microsoft's defined services, it is full of cloudcomputing potential that you can utilize in almost any way imaginable.

More companies than ever before are moving their data to cloud-based services. Knowing which provider to use can be difficult to determine. With these Microsoft Azure advantages and disadvantages, you'll get a better idea if this PaaS platform is capable of meeting your current and future needs.

\section{SUGGESTION}

The suggestions that can be given in connection with the implementation of this research are:
1. In its development, cloud infrastructure can be built on multi servers.

2. Cloud computing technology can be more optimized for organizations that have a small budget.

3. The use of cloud computing technology can also be used for universities for student research needs.

\section{REFERENCES}

[1] https://dailysocial.id/post/cloud-untuk-bisnis-online

[2] https://ccbtechnology.com/what-microsoft-azure-is-andwhy-it-matters/

[3] https://blog.5nine.com/what-is-microsoft-azure

[4] Azure_Developer_Guide_eBook_id-ID.

[5] ANALISIS_IMPLEMENTASI_INFRASTRUCTURE_AS 